\title{
Positive solutions for a class of superlinear semipositone systems on exterior domains
}

\author{
Abraham Abebe ${ }^{1}$, Maya Chhetri ${ }^{1 *}$, Lakshmi Sankar ${ }^{2}$ and R Shivaji ${ }^{1}$
}

*Correspondence: maya@uncg.edu 1 Department of Mathematics and Statistics, The University of North Carolina at Greensboro, Greensboro, USA

Full list of author information is available at the end of the article

\begin{abstract}
We study the existence of a positive radial solution to the nonlinear eigenvalue problem $-\Delta u=\lambda K_{1}(|x|) f(v)$ in $\Omega_{e},-\Delta v=\lambda K_{2}(|x|) g(u)$ in $\Omega_{e}, u(x)=v(x)=0$ if $|x|=r_{0}$ $(>0), u(x) \rightarrow 0, v(x) \rightarrow 0$ as $|x| \rightarrow \infty$, where $\lambda>0$ is a parameter, $\Delta u=\operatorname{div}(\nabla u)$ is the Laplace operator, $\Omega_{e}=\left\{x \in \mathbb{R}^{n}|| x \mid>r_{0}, n>2\right\}$, and $K_{i} \in C^{1}\left(\left[r_{0}, \infty\right),(0, \infty)\right) ; i=1,2$ are such that $K_{i}(|x|) \rightarrow 0$ as $|x| \rightarrow \infty$. Here $f, g:[0, \infty) \rightarrow \mathbb{R}$ are $C^{1}$ functions such that they are negative at the origin (semipositone) and superlinear at infinity. We establish the existence of a positive solution for $\lambda$ small via degree theory and rescaling arguments. We also discuss a non-existence result for $\lambda \gg 1$ for the single equations case.
\end{abstract}

MSC: $34 \mathrm{~B} 16 ; 34 \mathrm{~B} 18$

Keywords: superlinear; semipositone; positive solutions; existence; non-existence; exterior domains

\section{Introduction}

We consider the nonlinear elliptic boundary value problem

$$
\left.\begin{array}{ll}
-\Delta u=\lambda K_{1}(|x|) f(v) & \text { in } \Omega_{e}, \\
-\Delta v=\lambda K_{2}(|x|) g(u) & \text { in } \Omega_{e}, \\
u(x)=v(x)=0 & \text { if }|x|=r_{0}(>0), \\
u(x) \rightarrow 0, v(x) \rightarrow 0 & \text { as }|x| \rightarrow \infty,
\end{array}\right\}
$$

where $\lambda>0$ is a parameter, $\Delta u=\operatorname{div}(\nabla u)$ is the Laplace operator, and $\Omega_{e}=\left\{x \in \mathbb{R}^{n}|| x \mid>\right.$ $\left.r_{0}, n>2\right\}$ is an exterior domain. Here the nonlinearities $f, g:[0, \infty) \rightarrow \mathbb{R}$ are $C^{1}$ functions which satisfy:

$\left(\mathrm{H}_{1}\right) f(0)<0$ and $g(0)<0$ (semipositone).

$\left(\mathrm{H}_{2}\right)$ For $i=1,2$ there exist $b_{i}>0$ and $q_{i}>1$ such that $\lim _{s \rightarrow \infty} \frac{f(s)}{s^{q_{1}}}=b_{1}$, and $\lim _{s \rightarrow \infty} \frac{g(s)}{s^{q_{2}}}=b_{2}$.

Further, for $i=1,2$, the weight functions $K_{i} \in C^{1}\left(\left[r_{0}, \infty\right),(0, \infty)\right)$ are such that $K_{i}(|x|) \rightarrow 0$ as $|x| \rightarrow \infty$. In particular, we are interested in the challenging case, where $K_{i}$ do not decay too fast. Namely, we assume

$\left(\mathrm{H}_{3}\right)$ There exist $\tilde{d}_{1}>0, \tilde{d}_{2}>0, \rho \in(0, n-2)$ such that for $i=1,2$

$$
\frac{\tilde{d}_{1}}{|x|^{n+\rho}} \leq K_{i}(|x|) \leq \frac{\tilde{d}_{2}}{|x|^{n+\rho}} \quad \text { for }|x| \gg 1
$$

(c) 2014 Abebe et al.; licensee Springer. This is an Open Access article distributed under the terms of the Creative Commons Attribution License (http://creativecommons.org/licenses/by/4.0), which permits unrestricted use, distribution, and reproduction in any medium, provided the original work is properly credited. 
We then establish the following.

Theorem 1.1 Let $\left(\mathrm{H}_{1}\right)-\left(\mathrm{H}_{3}\right)$ hold. Then (1.1) has a positive radial solution $(u, v)(u>0$, $v>0$ in $\Omega_{e}$ ) when $\lambda$ is small, and $\|u\|_{\infty} \rightarrow \infty,\|v\|_{\infty} \rightarrow \infty$ as $\lambda \rightarrow 0$.

We prove this result via the Leray-Schauder degree theory, by arguments similar to those used in [1] and [2]. The study of such eigenvalue problems with semipositone structure has been documented to be mathematically challenging (see $[3,4])$, yet a rich history is developing starting from the 1980s (see [5-7]) until recently (see [8-12]). In [1, 2] the authors studied such superlinear semipositone problems on bounded domains. In particular, in [12] the authors studied the system

$$
\left.\begin{array}{ll}
-\Delta u=\lambda f(v) & \text { in } \Omega, \\
-\Delta v=\lambda g(u) & \text { in } \Omega, \\
u=v=0 & \text { on } \partial \Omega,
\end{array}\right\}
$$

where $\Omega$ is a bounded domain in $\mathbb{R}^{n}, n \geq 1$, and establish an existence result when $\lambda$ is small. The main motivation of this paper is to extend this study in the case of exterior domains (see Theorem 1.1).

We also discuss a non-existence result for the single equation model:

$$
\left.\begin{array}{ll}
-\Delta u=\lambda K_{1}(|x|) \tilde{f}(u) & \text { in } \Omega_{e}, \\
u(x)=0 & \text { if }|x|=r_{0}(>0), \\
u(x) \rightarrow 0 & \text { as }|x| \rightarrow \infty,
\end{array}\right\}
$$

for large values of $\lambda$, when $\tilde{f}, K_{1}$ satisfy the following hypotheses:

$\left(\mathrm{H}_{4}\right) \tilde{f} \in C^{1}([0, \infty), \mathbb{R}), \tilde{f}^{\prime}(z)>0$ for all $z>0, \tilde{f}(0)<0$, and there exists $m_{0}>0$ such that $\lim _{z \rightarrow \infty} \frac{\tilde{f}(z)}{z} \geq m_{0}$.

$\left(\mathrm{H}_{5}\right)$ The weight function $K_{1} \in C^{1}\left(\left[r_{0}, \infty\right),(0, \infty)\right)$ is such that $s^{\frac{-2(n-1)}{n-2}} K_{1}\left(r_{0} s^{\frac{1}{2-n}}\right)$ is decreasing for $s \in(0,1]$.

We establish the following.

Theorem 1.2 Let $\left(\mathrm{H}_{3}\right)-\left(\mathrm{H}_{5}\right)$ hold. Then (1.2) has no nonnegative radial solution for $\lambda \gg 1$.

We establish Theorem 1.2 by recalling various useful properties of solutions established in [13], where the authors prove a uniqueness result for $\lambda \gg 1$ for such an equation in the case when $\tilde{f}$ is sublinear at $\infty$. However, the properties we recall from [13] are independent of the growth behavior of $\tilde{f}$ at $\infty$. Non-existence results for such superlinear semipositone problems on bounded domain also have a considerable history starting from the work in the 1980s in [14] leading to the recent work in [15]. Here we discuss such a result for the first time on exterior domains. 
Finally, we note that the study of radial solutions $(u(r), v(r))$ (with $r=|x|)$ of (1.1) corresponds to studying

$$
\left.\begin{array}{ll}
-\left(r^{n-1} u^{\prime}(r)\right)^{\prime}=\lambda r^{n-1} K_{1}(r) f(v(r)) & \text { for } r>r_{0}, \\
-\left(r^{n-1} v^{\prime}(r)\right)^{\prime}=\lambda r^{n-1} K_{2}(r) g(u(r)) & \text { for } r>r_{0}, \\
u(r)=v(r)=0 & \text { if } r=r_{0}(>0), \\
u(r) \rightarrow 0, v(r) \rightarrow 0 & \text { as } r \rightarrow \infty,
\end{array}\right\}
$$

which can be reduced to the study of solutions $(u(s), v(s)) ; s \in[0,1]$ to the singular system:

$$
\left.\begin{array}{ll}
-u^{\prime \prime}(s)=\lambda h_{1}(s) f(v(s)), & 0<s<1, \\
-v^{\prime \prime}(s)=\lambda h_{2}(s) g(u(s)), & 0<s<1, \\
u(0)=u(1)=0, v(0)=v(1)=0, &
\end{array}\right\}
$$

via the Kelvin transformation $s=\left(\frac{r}{r_{0}}\right)^{2-n}$, where $h_{i}(s)=\frac{r_{0}^{2}}{(n-2)^{2}} s^{\frac{-2(n-1)}{(n-2)}} K_{i}\left(r_{0} s^{\frac{1}{2-n}}\right), i=1,2$ (see [16]).

Remark 1.3 The assumption $\left(\mathrm{H}_{3}\right)$ implies that $\lim _{s \rightarrow 0^{+}} h_{i}(s)=\infty$, for $i=1,2$, $\hat{h}=\inf _{t \in(0,1)}\left\{h_{1}(t), h_{2}(t)\right\}>0$, and there exist $d>0, \eta \in(0,1)$ such that $h_{i}(s) \leq \frac{d}{s^{\eta}}$ for $s \in(0,1]$, and for $i=1,2$. When in addition $\left(\mathrm{H}_{5}\right)$ is satisfied, $h_{1}$ is decreasing in $(0,1]$.

We will prove Theorem 1.1 in Section 2 by studying the singular system (1.3), and Theorem 1.2 in Section 3 by studying the corresponding single equation

$$
\left.\begin{array}{l}
-u^{\prime \prime}(s)=\lambda h_{1}(s) \tilde{f}(u(s)), \quad 0<s<1, \\
u(0)=u(1)=0
\end{array}\right\}
$$

\section{Existence result}

We first establish some useful results for solutions to the system

$$
\left.\begin{array}{ll}
-u^{\prime \prime}(s)=b_{1} h_{1}(s)|v(s)+l|^{q_{1}}, & 0<s<1, \\
-v^{\prime \prime}(s)=b_{2} h_{2}(s)|u(s)+l|^{q_{2}}, & 0<s<1, \\
u(0)=u(1)=0, v(0)=v(1)=0, &
\end{array}\right\}
$$

where $l \geq 0$ is a parameter. (Clearly, any solution $\left(u_{l}, v_{l}\right)$ of $(2.1)$ for $l>0$ must satisfy $u_{l}(s)>0, v_{l}(s)>0$ for $s \in(0,1)$. This is also true for any nontrivial solution when $l=0$.) We prove the following.

\section{Lemma 2.1}

(i) There exists $l_{0}>0$ such that 2.1 has no solution if $l \geq l_{0}$.

(ii) For each $l \in\left[0, l_{0}\right)$, there exists $M>0$ (independent of $l$ ) such that if $\left(u_{l}, v_{l}\right)$ is a solution of (2.1), then $\max \left\{\left\|u_{l}\right\|_{\infty},\left\|v_{l}\right\|_{\infty}\right\} \leq M$.

Proof of (i) Let $\lambda_{1}:=\pi^{2}, \phi_{1}:=\sin (\pi s)$. Here $\lambda_{1}$ is the principal eigenvalue and $\phi_{1}$ a corresponding eigenfunction of $-\phi^{\prime \prime}(s)=\lambda \phi(s)$ in $(0,1)$ with $\phi(0)=0=\phi(1)$. Let $a>\frac{\lambda_{1}}{\sqrt{b_{1} b_{2}} \hat{h}}, c>0$ be such that $(s+l)^{q_{i}} \geq a s-c$ for all $s \geq 0$ and for $i=1,2$. Now let $\left(u_{l}, v_{l}\right)$ be a solution of 
(2.1). Multiplying (2.1) by $\phi_{1}$ and integrating, we obtain

$$
\lambda_{1} \int_{0}^{1} u_{l} \phi_{1} d s=b_{1} \int_{0}^{1} h_{1}(s)\left(v_{l}+l\right)^{q_{1}} \phi_{1} d s \geq b_{1} \int_{0}^{1} h_{1}(s)\left(a v_{l}-c\right) \phi_{1} d s
$$

and

$$
\lambda_{1} \int_{0}^{1} v_{l} \phi_{1} d s=b_{2} \int_{0}^{1} h_{2}(s)\left(u_{l}+l\right)^{q_{2}} \phi_{1} d s \geq b_{2} \int_{0}^{1} h_{2}(s)\left(a u_{l}-c\right) \phi_{1} d s .
$$

By Remark 1.3, $\hat{h}=\inf _{t \in(0,1)}\left\{h_{1}(t), h_{2}(t)\right\}>0$, and $\left\|h_{i}\right\|_{1}:=\int_{0}^{1} h_{i}(s) d s<\infty$ for $i=1,2$. Then from the above inequalities we obtain

$$
\int_{0}^{1} v_{l} \phi_{1} d s \leq \frac{1}{a b_{1} \hat{h}}\left(\lambda_{1} \int_{0}^{1} u_{l} \phi_{1} d s+b_{1} c\left\|h_{1}\right\|_{1}\right)
$$

and

$$
\int_{0}^{1} u_{l} \phi_{1} d s \leq \frac{1}{a b_{2} \hat{h}}\left(\lambda_{1} \int_{0}^{1} v_{l} \phi_{1} d s+b_{2} c\left\|h_{2}\right\|_{1}\right)
$$

Hence we deduce that

$$
\int_{0}^{1} u_{l} \phi_{1} d s \leq \frac{m_{1}}{m}:=m_{2}
$$

where $m:=\left(a b_{2} \hat{h}-\frac{\lambda_{1}^{2}}{a b_{1} \hat{h}}\right)$, and $m_{1}:=\frac{\lambda_{1} c\left\|h_{1}\right\|_{1}}{a \hat{h}}+b_{2} c\left\|h_{2}\right\|_{1}$. This implies

$$
\int_{0}^{1}\left(v_{l}+l\right)^{q_{1}} \phi_{1} d s \leq \frac{\lambda_{1} m_{2}}{b_{1} \hat{h}}:=m_{3}
$$

In particular, this implies $\int_{\frac{1}{4}}^{\frac{3}{4}} l^{q_{1}} \mathrm{~d} s \leq \frac{m_{3}}{\inf _{\left[\frac{1}{4}, \frac{3}{4}\right]} \phi_{1}}$. Since $m_{3}$ is independent of $l$, clearly this is a contradiction for $l \gg 1$, and hence there must exists an $l_{0}>0$ such that for $l \geq l_{0},(2.1)$ has no solution.

Proof of (ii) Assume the contrary. Then without loss of generality we can assume there exists $\left\{l_{n}\right\} \subset\left(0, l_{0}\right)$ such that $\left\|u_{l_{n}}\right\|_{\infty} \rightarrow \infty$ as $n \rightarrow \infty$. Clearly $u_{l_{n}}^{\prime \prime}(s)<0$, and $v_{l_{n}}^{\prime \prime}(s)<0$ for all $s \in(0,1)$. Let $s_{1\left(l_{n}\right)} \in(0,1), s_{2\left(l_{n}\right)} \in(0,1)$ be the points at which $u_{l_{n}}$ and $v_{l_{n}}$ attain their maximums. Now since $u_{l_{n}}^{\prime \prime}(s)<0$ for all $s \in(0,1)$, we have

$$
u_{l_{n}}(s) \geq \begin{cases}\frac{s u_{l_{n}}\left(s_{1\left(l_{n}\right)}\right)}{s_{1}\left(l_{n}\right)} & \text { for } s \in\left(0, s_{1\left(l_{n}\right)}\right), \\ \frac{(1-s) u_{l_{n}}\left(s_{1\left(l_{n}\right)}\right)}{1-s_{1\left(l_{n}\right)}} & \text { for } s \in\left(s_{1\left(l_{n}\right)}, 1\right) .\end{cases}
$$

Hence $u_{l_{n}}(s) \geq \min \left\{\frac{s\left\|u_{l_{n}}\right\|_{\infty}}{s_{1\left(l_{n}\right)}}, \frac{(1-s)\left\|u_{l_{n}}\right\|_{\infty}}{1-s_{1\left(l_{n}\right)}}\right\}$, and in particular, for $s \in\left[\frac{1}{4}, \frac{3}{4}\right]$,

$$
u_{l_{n}}(s) \geq \min \left\{\frac{1}{4}\left\|u_{l_{n}}\right\|_{\infty}, \frac{1}{4}\left\|u_{l_{n}}\right\|_{\infty}\right\}=\frac{1}{4}\left\|u_{l_{n}}\right\|_{\infty} .
$$


Let $\tilde{s_{l_{n}}}, \overline{s_{l_{n}}} \in\left[\frac{1}{4}, \frac{3}{4}\right]$ be such that $\min _{\left[\frac{1}{4}, \frac{3}{4}\right]} u_{l_{n}}(s)=u_{l_{n}}\left(\tilde{s_{l_{n}}}\right)$, and $\min _{\left[\frac{1}{4}, \frac{3}{4}\right]} v_{l_{n}}(s)=v_{l_{n}}\left(\overline{s_{l_{n}}}\right)$. Now for $s \in\left[\frac{1}{4}, \frac{3}{4}\right]$,

$$
v_{l_{n}}(s) \geq b_{2} \hat{h} \tilde{m} \int_{\frac{1}{4}}^{\frac{3}{4}}\left|u_{l_{n}}(t)+l\right|^{q_{2}} d t
$$

where $\tilde{m}:=\min _{\left[\frac{1}{4}, \frac{3}{4}\right] \times\left[\frac{1}{4}, \frac{3}{4}\right]} G(s, t)(>0)$, and $G$ is the Green's function of $-Z^{\prime \prime}$ with $Z(0)=0=$ $Z(1)$. In particular, $v_{l_{n}}\left(\overline{l_{n}}\right) \geq b_{2} \hat{h} \frac{\tilde{m}}{2}\left(u_{l_{n}}\left(\tilde{s_{n}}\right)\right)^{q_{2}}$. Similarly $u_{l_{n}}\left(\tilde{s_{n}}\right) \geq b_{1} \hat{h} \frac{m}{2}\left(v_{l_{n}}\left(\overline{s_{n}}\right)\right)^{q_{1}}$. Hence, there exists a constant $A>0$ such that

$$
u_{l_{n}}\left(\tilde{s_{n}}\right) \geq A\left(u_{l_{n}}\left(\tilde{l_{n}}\right)\right)^{q_{1} q_{2}} .
$$

This is a contradiction since $q_{1} q_{2}>1$ and $u_{l_{n}}\left(\tilde{s_{n}}\right) \geq \frac{1}{4}\left\|u_{l_{n}}\right\|_{\infty} \rightarrow \infty$ as $n \rightarrow \infty$. Thus (ii) holds.

Proof of Theorem 1.1 We first extend $f$ and $g$ as even functions on $\mathbb{R}$ by setting $f(-s)=f(s)$ and $g(-s)=g(s)$. Then we use the rescaling, $\lambda=\gamma^{\delta}, w_{1}=\gamma u$, and $w_{2}=\gamma^{\theta} v$ with $\gamma>0$, $\theta=\frac{q_{2}+1}{q_{1}+1}$, and $\delta=\frac{q_{1} q_{2}-1}{q_{1}+1}$. With this rescaling, (1.3) reduces to

$$
\begin{aligned}
& -w_{1}^{\prime \prime}(s)=F\left(s, \gamma, w_{2}\right), \\
& -w_{2}^{\prime \prime}(s)=G\left(s, \gamma, w_{1}\right), \\
& w_{1}(0)=w_{1}(1)=0, w_{2}(0)=w_{2}(1)=0,
\end{aligned}
$$

where

$$
\begin{aligned}
& F\left(s, \gamma, w_{2}\right):=\gamma^{1+\delta} h_{1}(s)\left(f\left(\frac{w_{2}}{\gamma^{\theta}}\right)-b_{1}\left|\frac{w_{2}}{\gamma^{\theta}}\right|^{q_{1}}\right)+b_{1}\left|w_{2}\right|^{q_{1}} h_{1}(s), \quad \text { and } \\
& G\left(s, \gamma, w_{1}\right):=\gamma^{\theta+\delta} h_{2}(s)\left(g\left(\frac{w_{1}}{\gamma}\right)-b_{2}\left|\frac{w_{1}}{\gamma}\right|^{q_{2}}\right)+b_{2}\left|w_{1}\right|^{q_{2}} h_{2}(s) .
\end{aligned}
$$

Note that by our hypothesis $\left(\mathrm{H}_{2}\right), F\left(s, \gamma, w_{2}\right) \rightarrow b_{1}\left|w_{2}\right|^{q_{1}} h_{1}(s)$ and $G\left(s, \gamma, w_{1}\right) \rightarrow b_{2}\left|w_{1}\right|^{q_{2}} \times$ $h_{2}(s)$ as $\gamma \rightarrow 0$. Hence we can continuously extend $F\left(s, \gamma, w_{2}\right)$ and $G\left(s, \gamma, w_{1}\right)$ to $F\left(s, 0, w_{2}\right)=$ $b_{1}\left|w_{2}\right|^{q_{1}} h_{1}(s)$ and $G\left(s, 0, w_{1}\right)=b_{2}\left|w_{1}\right|^{q_{2}} h_{2}(s)$, respectively. Note that proving (1.3) has a positive solution for $\lambda$ small is equivalent to proving (2.2) has a solution $\left(w_{1}, w_{2}\right)$ with $w_{1}>0$, $w_{2}>0$ in $(0,1)$ for small $\gamma>0$. We will achieve this by establishing that the limiting equation (when $\gamma=0$ )

$$
\left.\begin{array}{ll}
-w_{1}^{\prime \prime}(s)=F\left(s, 0, w_{2}\right)=b_{1} h_{1}(s)\left|w_{2}\right|^{q_{1}}, & 0<s<1, \\
-w_{2}^{\prime \prime}(s)=G\left(s, 0, w_{1}\right)=b_{2} h_{2}(s)\left|w_{1}\right|^{q_{2}}, & 0<s<1, \\
w_{1}(0)=w_{1}(1)=0, w_{2}(0)=w_{2}(1)=0 &
\end{array}\right\}
$$

(which is the same as (2.1) with $l=0$ ) has a positive solution $w_{1}>0, w_{2}>0$ in $(0,1)$ that persists for small $\gamma>0$.

Let $X=C_{0}[0,1] \times C_{0}[0,1]$ be the Banach space equipped with $\|\underline{w}\|_{X}=\left\|\left(w_{1}, w_{2}\right)\right\|_{X}=$ $\max \left\{\left\|w_{1}\right\|_{\infty},\left\|w_{2}\right\|_{\infty}\right\}$, where $\|\cdot\|_{\infty}$ denotes the usual supremum norm in $C_{0}([0,1])$. Then for fixed $\gamma \geq 0$, we define the map $S(\gamma, \cdot): X \rightarrow X$ by

$$
S(\gamma, \underline{w}):=\underline{w}-\left(K\left(F\left(s, \gamma, w_{2}\right)\right), K\left(G\left(s, \gamma, w_{1}\right)\right)\right),
$$


where $K(H(s, \gamma, Z(s)))=\int_{0}^{1} G(t, s) H(t, \gamma, Z(t)) d t$. Note that $F(s, \gamma, \cdot), G(s, \gamma, \cdot): C_{0}([0,1]) \rightarrow$ $L^{1}(0,1)$ are continuous and $K: L^{1}(0,1) \rightarrow C_{0}^{1}([0,1])$ is compact. Hence $S(\gamma, \cdot)$ is a compact perturbation of the identity. Clearly for $\gamma>0$, if $S(\gamma, \underline{w})=\underline{0}$, then $\underline{w}=\left(w_{1}, w_{2}\right)$ is a solution of $(2.2)$, and if $S(0, \underline{w})=\underline{0}$, then $\underline{w}=\left(w_{1}, w_{2}\right)$ is a solution of $(2.3)$.

We first establish the following.

Lemma 2.2 There exists $R>0$ such that $S(0, \underline{w}) \neq \underline{0}$ for all $\underline{w}=\left(w_{1}, w_{2}\right) \in X$ with $\|\underline{w}\|_{X}=R$ and $\operatorname{deg}\left(S(0, \cdot), B_{R}(\underline{0}), \underline{0}\right)=0$.

Proof Define $S^{l}(0, \underline{w}): X \rightarrow X$ by

$$
S^{l}(0, \underline{w}):=\underline{w}-\left(K\left(b_{1} h_{1}(s)\left|w_{2}+l\right|^{q_{1}}\right), K\left(b_{2} h_{2}(s)\left|w_{1}+l\right|^{q_{2}}\right)\right)
$$

for $l \geq 0$. (Note $S^{0}(0, \underline{w})=S(0, \underline{w})$.) By Lemma 2.1, if $l \geq l_{0}$ then $S^{l}(0, \underline{w}) \neq \underline{0}$ and if $S^{l}(0, \underline{w})=\underline{0}$ for $l \in\left[0, l_{0}\right)$, then $\|w\|_{X} \leq M$. This implies that there exists $R \gg 1$ such that $S^{l}(0, \underline{w}) \neq \underline{0}$ for $\underline{w} \in \partial B_{R}(\underline{0})$ for any $l \geq 0$. Also, since (2.1) has no solution for $l \geq l_{0}$, $\operatorname{deg}\left(S^{l_{0}}(0, \cdot), B_{R}(\underline{0}), \underline{0}\right)=0$. Hence, using the homotopy invariance of degree with the parameter $l \in\left[0, l_{0}\right]$ we get

$$
\operatorname{deg}\left(S(0, \cdot), B_{R}(\underline{0}), \underline{0}\right)=\operatorname{deg}\left(S^{l_{0}}(0, \cdot), B_{R}(\underline{0}), \underline{0}\right)=0 \text {. }
$$

Next we establish the following.

Lemma 2.3 There exists $r \in(0, R)$ small enough such that $S(0, \underline{w}) \neq \underline{0}$ for all $\underline{w}=\left(w_{1}, w_{2}\right) \in$ $X$ with $\|\underline{w}\|_{X}=r$ and $\operatorname{deg}\left(S(0, \cdot), B_{r}(\underline{0}), \underline{0}\right)=1$.

Proof Define $T^{\tau}(0, \underline{w}): X \rightarrow X$ by

$$
T^{\tau}(0, \underline{w}):=\underline{w}-\left(K\left(\tau b_{1} h_{1}(s)\left|w_{2}\right|^{q_{1}}\right), K\left(\tau b_{2} h_{2}(s)\left|w_{1}\right|^{q_{2}}\right)\right)
$$

for $\tau \in[0,1]$. Clearly $T^{1}(0, \underline{w})=S(0, \underline{w})$, and $T^{0}(0, \underline{w})=I$ is the identity operator. Note that $T^{\tau}(0, \underline{w})=0$ if $\underline{w}=\left(w_{1}, w_{2}\right)$ is a solution of

$$
\left.\begin{array}{ll}
-w_{1}^{\prime \prime}(s)=\tau b_{1} h_{1}(s)\left|w_{2}\right|^{q_{1}}, & 0<s<1, \\
-w_{2}^{\prime \prime}(s)=\tau b_{2} h_{2}(s)\left|w_{1}\right|^{q_{2},} & 0<s<1, \\
w_{1}(0)=w_{1}(1)=0, w_{2}(0)=w_{2}(1)=0, &
\end{array}\right\}
$$

and for $\tau=1$, (2.4) coincides with (2.3). Assume to the contrary that (2.4) has a solution $\underline{w}=\left(w_{1}, w_{2}\right)$ with $\|\underline{w}\|_{X}=\tilde{r}>0$. Without loss of generality assume $\left\|w_{1}\right\|_{\infty}=\tilde{r}$. Now,

$$
w_{1}(s)=\tau \int_{0}^{1} G(s, t) b_{1} h_{1}(s)\left|w_{2}\right|^{q_{1}} d s .
$$

Then $\left\|w_{1}\right\|_{\infty} \leq \tilde{C}\left\|w_{2}\right\|_{\infty}^{q_{1}}$ for some constant $\tilde{C}>0$ independent of $\tau \in[0,1]$. Similarly $\left\|w_{2}\right\|_{\infty} \leq \hat{C}\left\|w_{1}\right\|_{\infty}^{q_{2}}$ for some constant $\hat{C}>0$. This implies that

$$
\tilde{r}=\left\|w_{1}\right\|_{\infty} \leq C\left\|w_{1}\right\|_{\infty}^{q_{1} q_{2}}=C \tilde{r}^{q_{1} q_{2}}
$$


for some constant $C>0$. But $q_{1} q_{2}>1$, and hence this is a contradiction if $\tilde{r}>0$ is small. Thus there exists small $r>0$ such that (2.4) has no solution $\underline{w}$ with $\|\underline{w}\|_{X}=r$ for all $\tau \in[0,1]$. Now using the homotopy invariance of degree with the parameter $\tau \in[0,1]$, in particular using the values $\tau=1$ and $\tau=0$, we obtain

$$
\operatorname{deg}\left(S(0, \cdot), B_{r}(\underline{0}), \underline{0}\right)=\operatorname{deg}\left(T^{1}(0, \cdot), B_{r}(\underline{0}), \underline{0}\right)=\operatorname{deg}\left(T^{0}(0, \cdot), B_{r}(\underline{0}), \underline{0}\right)=1 .
$$

By Lemma 2.2 and Lemma 2.3, with $0<r<R$, we conclude that

$$
\operatorname{deg}\left(S(0, \cdot), B_{R}(\underline{0}) \backslash \overline{B_{r}}(\underline{0}), \underline{0}\right)=-1,
$$

and hence (2.3) has a solution $\underline{w}=\left(w_{1}, w_{2}\right)$ with $w_{1}>0, w_{2}>0$ in $(0,1)$, and $r<\|w\|_{X}<R$. Now we show that the solution obtained above (when $\gamma=0$ ) persists for small $\gamma>0$ and remains positive componentwise.

Lemma 2.4 Let $R, r$ be as in Lemmas 2.2, 2.3, respectively. Then there exists $\gamma_{0}>0$ such that:

(i) $\operatorname{deg}\left(S(\gamma, \cdot), B_{R}(\underline{0}) \backslash \overline{B_{r}}(\underline{0}), \underline{0}\right)=-1$ for all $\gamma \in\left[0, \gamma_{0}\right]$.

(ii) If $S(\gamma, \underline{w})=\underline{0}$ for $\gamma \in\left[0, \gamma_{0}\right]$ with $r<\|\underline{w}\|_{X}<R$, then $w_{1}>0, w_{2}>0$ in $(0,1)$.

Proof of (i) We first show that there exists $\gamma_{0}>0$ such that $S(\gamma, \underline{w}) \neq \underline{0}$ for all $\underline{w}=\left(w_{1}, w_{2}\right) \in$ $X$ with $\|\underline{w}\|_{X} \in\{R, r\}$, for all $\gamma \in\left[0, \gamma_{0}\right]$. Suppose to the contrary that there exists $\left\{\gamma_{n}\right\}$ with $\gamma_{n} \rightarrow 0, S\left(\gamma_{n}, \underline{w_{n}}\right)=\underline{0}$ and $\left\|\underline{w_{n}}\right\|_{X} \in\{r, R\}$. Since $\underline{K}=(K, K): L^{1}(0,1) \times L^{1}(0,1) \rightarrow$ $C_{0}^{1}([0,1]) \times C_{0}^{1}([0,1])$ is compact, and $\left\{F\left(s, \gamma_{n}, w_{2 n}\right), G\left(s, \gamma_{n}, w_{1 n}\right)\right\}$ are bounded in $L^{1}(0,1) \times$ $L^{1}(0,1), \underline{w}_{n} \rightarrow \underline{Z}=\left(Z_{1}, Z_{2}\right) \in C_{0}^{1}([0,1]) \times C_{0}^{1}([0,1])$ (up to a subsequence) with $\|\underline{Z}\|_{X}=R$ or $r$ and $S(0, \underline{Z})=\underline{0}$. This is a contradiction to Lemma 2.2 or 2.3 and hence there exists a small $\gamma_{0}>0$ satisfying the assertions. Now, by the homotopy invariance of degree with respect to $\gamma \in\left[0, \gamma_{0}\right]$,

$$
\operatorname{deg}\left(S(\gamma, \cdot), B_{R}(\underline{0}) \backslash \overline{B_{r}}(\underline{0}), \underline{0}\right)=\operatorname{deg}\left(S(0, \cdot), B_{R}(\underline{0}) \backslash \overline{B_{r}}(\underline{0}), \underline{0}\right)=-1
$$

for all $\gamma \in\left[0, \gamma_{0}\right]$.

Proof of (ii) Assume to the contrary that there exists $\gamma_{n} \rightarrow 0$ and a corresponding solution $\underline{w_{n}}=\left(w_{1 n}, w_{2 n}\right)$ such that $r<\left\|\underline{w_{n}}\right\|_{X}<R$ and

$$
\Omega_{n}:=\left\{x \in(0,1) \mid w_{1 n}(x) \leq 0 \text { or } w_{2 n}(x) \leq 0\right\} \neq \emptyset .
$$

Arguing as before, $\underline{w_{n}} \rightarrow \underline{Z} \in C_{0}^{1}([0,1]) \times C_{0}^{1}([0,1])$ with $S(0, \underline{Z})=\underline{0}$ (up to a subsequence). Note that $\underline{Z} \not \equiv \underline{0}$ since $\|\underline{Z}\|_{X} \geq r>0$. By the strong maximum principle $Z_{1}>0, Z_{2}>0$, $Z_{1}^{\prime}(0)>0, Z_{2}^{\prime}(0)>0, Z_{1}^{\prime}(1)<0$ and $Z_{2}^{\prime}(1)<0$. Now suppose there exists $\left\{x_{n}\right\} \in(0,1)$ with $\left\{x_{n}\right\} \in \Omega_{n}$ and $w_{1 n}\left(x_{n}\right) \leq 0$. Then $\left\{x_{n}\right\}$ must have a subsequence (renamed as $\left\{x_{n}\right\}$ itself) such that $x_{n} \rightarrow \tilde{x} \in[0,1]$. But $Z_{1}>0$ in $(0,1)$ implies that $\tilde{x} \in\{0,1\}$. Suppose $\tilde{x}=0$. Since $w_{1 n}\left(x_{n}\right) \leq 0$ and $w_{1 n}(0)=0$, there exists $y_{n} \in\left(0, x_{n}\right)$ such that $w_{1 n}^{\prime}\left(y_{n}\right) \leq 0$, and hence taking the limit as $n \rightarrow \infty$ we will have $Z_{1}^{\prime}(0) \leq 0$, which is a contradiction since $Z_{1}^{\prime}(0)>0$. A similar contradiction follows if $\tilde{x}=1$, using the fact that $Z_{1}^{\prime}(1)<0$. Further, contradictions can 
be achieved if there exists $\left\{x_{n}\right\} \in \Omega$ with $\left\{x_{n}\right\} \in \Omega_{n}$ and $w_{2 n}\left(x_{n}\right) \leq 0$ using the facts that $Z_{2}^{\prime}(0)>0$ and $Z_{2}^{\prime}(1)<0$. This completes the proof of the lemma.

We now easily conclude the proof of Theorem 1.1 . From Lemma 2.4 , since $\underline{w}=\left(w_{1}, w_{2}\right)$ is a positive solution of (2.2) for $\gamma$ small, $(u, v)=\left(\gamma^{-1} w_{1}, \gamma^{-\theta} w_{2}\right)$ with $\theta=\frac{q_{2}+1}{q_{1}+1}$ is a positive solution of (1.3) for $\lambda=\gamma^{\delta}$ where $\delta=\frac{q_{1} q_{2}-1}{q_{1}+1}$. Further, since $w_{1}>0$ and $w_{2}>0$ in $(0,1)$ for $\gamma \in\left[0, \gamma_{0}\right],\|u\|_{\infty} \rightarrow \infty$ and $\|v\|_{\infty} \rightarrow \infty$ as $\lambda\left(=\gamma^{\delta}\right) \rightarrow 0$. This completes the proof of Theorem 1.1.

\section{Non-existence result}

We first recall from [13] that, when $\left(\mathrm{H}_{5}\right)$ is satisfied, one can prove via an energy analysis that a nonnegative solution $u$ of $(1.4)$ must be positive in $(0,1)$ and have a unique interior maximum with maximum value greater than $\theta$, where $\theta$ is the unique positive zero of $\tilde{F}(s)=\int_{0}^{s} \tilde{f}(y) d y$. Further, for $\lambda \gg 1$ and $s_{1}, \hat{s_{1}} \in(0,1)$ such that $\hat{s_{1}}>s_{1}, u\left(s_{1}\right)=u\left(\hat{s_{1}}\right)=\beta$ (see Figure 1), where $\beta>0$ is the unique zero of $\tilde{f}$, there exists a constant $C$ such that $s_{1} \leq C \lambda^{-\frac{1}{2}}$ and $\left(1-\hat{s_{1}}\right) \leq C \lambda^{-\frac{1}{2}}$. Hence we can assume $\left(\hat{s_{1}}-s_{1}\right)>\frac{1}{2}$ for $\lambda \gg 1$. Now we provide the proof of Theorem 1.2.

Proof of Theorem 1.2 Let $v:=u-\beta$. Then $v>0$ in $\left(s_{1}, \hat{s_{1}}\right)$ and satisfies

$$
\left.\begin{array}{l}
-v^{\prime \prime}=\lambda h_{1}(s) \frac{\tilde{f}(u)}{u-\beta} \nu, \quad s_{1}<s<\hat{s_{1}} \\
v\left(s_{1}\right)=v\left(\hat{s_{1}}\right)=0 .
\end{array}\right\}
$$

Note that $\phi(s)=-\left(\sin \left(\frac{\pi\left(s-s_{1}\right)}{\left(\hat{s_{1}}-s_{1}\right)}\right)\right)>0$ in $\left(s_{1}, \hat{s_{1}}\right), \phi\left(s_{1}\right)=\phi\left(\hat{s_{1}}\right)=0$, and it satisfies $-\phi^{\prime \prime}=\frac{\pi^{2}}{\left(\hat{s_{1}}-s_{1}\right)^{2}} \phi$ in $\left(s_{1}, \hat{s_{1}}\right)$. Hence using the fact that $\int_{s_{1}}^{s_{1}}\left(-\phi v^{\prime \prime}+\nu \phi^{\prime \prime}\right) d s=0$, we obtain

$$
\int_{s_{1}}^{\hat{s_{1}}}\left(\lambda \frac{\tilde{f}(u)}{u-\beta} h_{1}(s)-\frac{\pi^{2}}{\left(\hat{s_{1}}-s_{1}\right)^{2}}\right) \nu \phi d s=0 .
$$

In particular,

$$
\lambda \frac{\tilde{f}\left(u\left(s_{\lambda}\right)\right)}{u\left(s_{\lambda}\right)-\beta} h_{1}\left(s_{\lambda}\right)=\frac{\pi^{2}}{\left(\hat{s_{1}}-s_{1}\right)^{2}}, \quad \text { for some } s_{\lambda} \in\left(s_{1}, \hat{s_{1}}\right)
$$

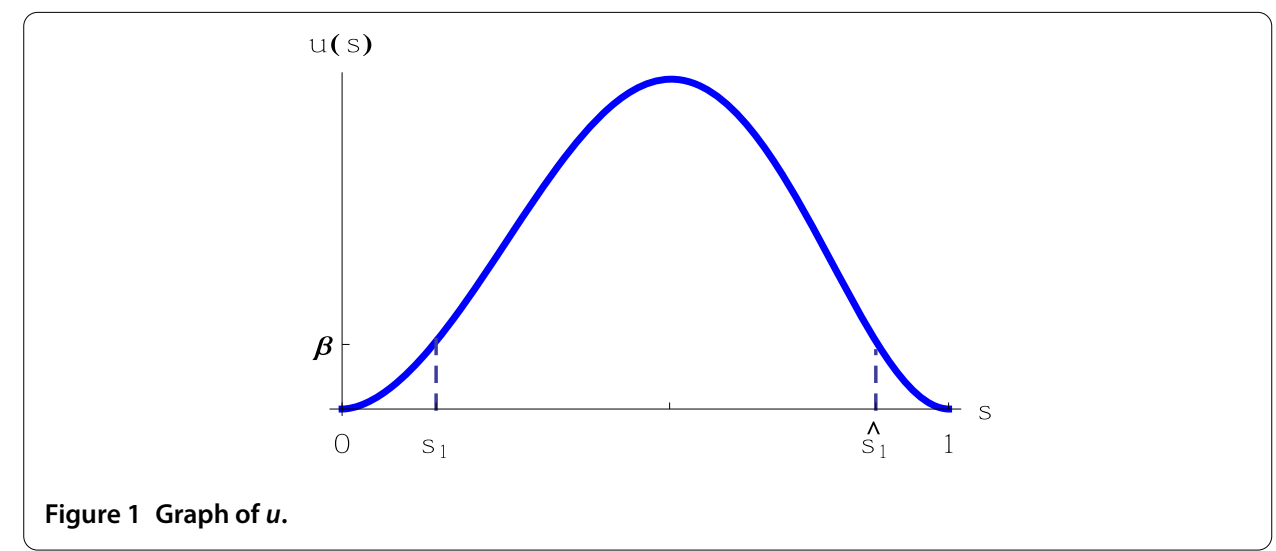


But $\hat{h}=\inf _{(0,1)} h_{1}(s)>0$, and $\left(\hat{s}_{1}-s_{1}\right)>\frac{1}{2}$ for $\lambda \gg 1$. Thus clearly (3.1) can hold when $\lambda \rightarrow \infty$, only if $Z=u\left(s_{\lambda}\right) \rightarrow \infty$ with $\frac{\tilde{f}\left(u\left(s_{\lambda}\right)\right)}{u\left(s_{\lambda}\right)-\beta} \rightarrow 0$. But by $\left(\mathrm{H}_{4}\right)$, this is not possible since $\lim _{Z \rightarrow \infty} \frac{\tilde{f}(Z)}{Z} \geq m_{0}>0$. Hence the nonnegative solution cannot exist for $\lambda \gg 1$.

\section{Competing interests}

The authors declare that they have no competing interests.

\section{Authors' contributions}

All authors contributed equally to the writing of this paper. All authors read and approved the final manuscript.

\section{Author details}

'Department of Mathematics and Statistics, The University of North Carolina at Greensboro, Greensboro, USA.

${ }^{2}$ Department of Mathematics \& NTIS, University of West Bohemia, Univerzitní 22, Plzeň, 30614, Czech Republic.

\section{Acknowledgements}

The third author is funded by the project EXLIZ - CZ.1.07/2.3.00/30.0013, which is co-financed by the European Social Fund and the state budget of the Czech Republic.

Received: 8 May 2014 Accepted: 5 August 2014 Published online: 25 September 2014

\section{References}

1. Ambrosetti, A, Arcoya, D, Buffoni, B: Positive solutions for some semi-positone problems via bifurcation theory. Differ. Integral Equ. 7(3-4), 655-663 (1994)

2. Maya, C, Girg, P: Existence and nonexistence of positive solutions for a class of superlinear semipositone systems. Nonlinear Anal. 71(10), 4984-4996 (2009)

3. Berestycki, H, Caffarelli, LA, Nirenberg, L: Inequalities for second-order elliptic equations with applications to unbounded domains. I. Duke Math. J. 81(2), 467-494 (1996). A celebration of John F. Nash, Jr

4. Lions, P-L: On the existence of positive solutions of semilinear elliptic equations. SIAM Rev. 24(4), $441-467$ (1982)

5. Brown, KJ, Shivaji, R: Simple proofs of some results in perturbed bifurcation theory. Proc. R. Soc. Edinb., Sect. A 93(1-2), 71-82 (1982/1983)

6. Castro, A, Shivaji, R: Nonnegative solutions for a class of nonpositone problems. Proc. R. Soc. Edinb., Sect. A 108(3-4), 291-302 (1988)

7. Castro, A, Shivaji, R: Nonnegative solutions to a semilinear Dirichlet problem in a ball are positive and radially symmetric. Commun. Partial Differ. Equ. 14(8-9), 1091-1100 (1989)

8. Hai, DD, Sankar, L, Shivaji, R: Infinite semipositone problems with asymptotically linear growth forcing terms. Differ. Integral Equ. 25(11-12), 1175-1188 (2012)

9. Lee, EK, Sankar, L, Shivaji, R: Positive solutions for infinite semipositone problems on exterior domains. Differ. Integral Equ. 24(9-10), 861-875 (2011)

10. Lee, EK, Shivaji, R, Ye, J: Classes of infinite semipositone systems. Proc. R. Soc. Edinb., Sect. A 139(4), $853-865$ (2009)

11. Sankar, L, Sasi, S, Shivaji, R: Semipositone problems with falling zeros on exterior domains. J. Math. Anal. Appl. 401(1), 146-153 (2013)

12. Maya, C, Girg, P: Existence of positive solutions for a class of superlinear semipositone systems. J. Math. Anal. Appl. 408(2), 781-788 (2013)

13. Castro, A, Sankar, L, Shivaji, R: Uniqueness of nonnegative solutions for semipositone problems on exterior domains. J. Math. Anal. Appl. 394(1), 432-437 (2012)

14. Brown, KJ, Castro, A, Shivaji, R: Nonexistence of radially symmetric nonnegative solutions for a class of semi-positone problems. Differ. Integral Equ. 2(4), 541-545 (1989)

15. Shivaji, R, Ye, J: Nonexistence results for classes of $3 \times 3$ elliptic systems. Nonlinear Anal. 74(4), 1485-1494 (2011)

16. Ko, E, Lee, EK, Shivaji, R: Multiplicity results for classes of singular problems on an exterior domain. Discrete Contin. Dyn. Syst. 33(11-12), 5153-5166 (2013) 